\title{
A SAÚDE DO TRABALHADOR NA SOCIEDADE 24 HORAS
}

\author{
Claudia Roberta de Castro Moreno \\ Frida Marina Fischer \\ LÚCia RotenBerg
}

\begin{abstract}
Resumo: Este artigo trata, em um primeiro momento, de fatores subjacentes às diferenças individuais quanto à tolerância ao trabalho em turnos e noturno. Associadas a esses fatores, também são apresentadas características do trabalho que podem ou não favorecer a tolerância ao trabalho em turnos. Em um segundo momento, apresenta-se medidas de intervenção que visam minimizar as dificuldades enfrentadas pelos trabalhadores quanto à saúde e ao bem-estar orgânico e social.

Palavras-chave: trabalho em turnos; organização do trabalho; ergonomia.

Abstract: This article first addresses the underlying factors determining the varying tolerance of individuals to swing and night shifts. Also discussed are features of specific jobs that may or may not favor the tolerance to variable and evening shifts. The second part of this article proposed measures to improve workers' health and physical and social well-being.

Key words: shift work; organization of labor; ergometrics.
\end{abstract}

A tualmente, existem no Brasil cerca de 64 milhões de pessoas com 10 anos ou mais ocupadas em vários tipos de trabalho, conforme dados obtidos pelo recenseamento realizado no ano $2000 .{ }^{1}$ Quase a metade dessa população (cerca de 28 milhões) trabalha mais que as 44 horas semanais, previstas na Constituição de 1988 como a jornada máxima de trabalho semanal. Para que essa jornada semanal seja cumprida, parece bastante razoável supor que, pelo menos no caso de parte desses trabalhadores, o trabalho seja exercido além do horário diurno. Há, portanto, uma parcela da população economicamente ativa que, além de trabalhar mais que o número de horas semanais previstas em lei, ainda o faz em horário noturno.

Adiciona-se a essa parcela de trabalhadores os que, embora não trabalhem mais que 44 horas semanais, o fazem em horários não usuais e obtém-se o número de trabalhadores em turnos e noturnos da população brasileira. Infelizmente, não há dados oficiais sobre o tamanho dessa população em nível nacional. Em 1994, levantamento da Fundação SEADE na área metropolitana de São Paulo caracterizou como trabalhador em turnos ou noturno 8,6\% da população (Fischer et al., 1995). Valendo-se desses dados, estima-se haver cerca de $10 \%$ da população brasileira ativa que trabalha em turnos ou à noite. Acredita-se que essa porcentagem seja até maior, uma vez que o oferecimento de serviços disponíveis muitas horas por dia, durante os dias de semana e fins de semana, vêm aumentando nos últimos anos. Pode-se citar, como exemplos, todos os serviços de telecomunicações, de processamento bancário, de distribuição de correspondência rápida, os centros de compras (shopping centers, supermercados), hotéis, lazer (cinemas, restaurantes, academias de ginástica, clubes sociais e esportivos), serviços educacionais. Em outras palavras, além dos serviços essenciais há uma quantidade cada vez maior de produção de bens e prestação de serviços que funcionam ininterruptamente. Para que esses bens sejam produzidos e os serviços prestados vem ocorrendo aumento da população que trabalha em turnos, em horário noturno ou em horários irregulares.

A demógrafa americana Harriet Presser (1999), ao apontar os efeitos do trabalho em turnos na sociedade, comenta os principais fatores que influenciaram o aumento do trabalho realizado além dos tradicionais horários diurnos e nos fins de semana: as rápidas mudanças que ocorreram nos processos tecnológicos, as características demográficas das po- 
pulações, a globalização econômica. Esse último fator levou a um grande incremento de atividades no setor de serviços, particularmente nas empresas que utilizam computadores em rede para manter seus negócios. $\mathrm{O}$ e-business criado com os serviços da Internet, bem como o aumento das corporações internacionais que têm escritórios e serviços em vários países do mundo, foi um importante passo para a expansão do trabalho não diurno, e em dias tradicionalmente dedicados ao descanso semanal.

Não há, portanto, como negar a existência de uma "sociedade 24 horas", a qual depende de vasto número de trabalhadores, que estão, por sua vez, sujeitos à exposição de fatores psicossociais do trabalho que interferem nos processos saúde-doença.

Neste artigo, serão abordados alguns desses fatores, seus efeitos na saúde dos trabalhadores, assim como as principais medidas de intervenção que visam minimizar as dificuldades enfrentadas pelos trabalhadores quanto à saúde e ao bem-estar orgânico e social.

\section{FATORES SUBJACENTES ÀS DIFERENÇAS INDIVIDUAIS QUANTO À TOLERÂNCIA AO TRABALHO EM TURNOS E NOTURNO}

As condições de trabalho e a organização do trabalho influenciam de forma significativa a tolerância ao trabalho em turnos e noturno. Particularmente, trabalhar em horários não diurnos pode levar os trabalhadores a ter pior desempenho em suas tarefas, a expô-los a maiores riscos de acidentes de trabalho e, de forma mais acentuada, a estressores ambientais, que podem levá-los à incapacidade funcional precoce.

\section{Condições de Trabalho}

Há vários séculos, já haviam sido estabelecidas jornadas de trabalho diurna e noturna, em especial nas atividades industriais, extrativas e dos serviços de saúde. Em 1556, o médico Georg Bauer descreve, em seu livro De Re Metallica, as dificuldades pelas quais passavam os mineiros do terceiro turno (o turno da noite) (Hunter, 1975). Escrito há 447 anos, este livro relata uma situação que ainda ocorre nos dias de hoje: o trabalho noturno combinado com vários outros estressores ambientais. Nos tempos atuais, as empresas empenham-se em bons resultados nos custos de produção, preocupam-se com a obsolescência técnica dos equipamentos e implantam o trabalho em turnos, incluindo as jornadas noturnas, sempre que as razões técnicas e econômicas se manifestarem (Rutenfranz et al., 1989).

As escalas de trabalho em turnos geralmente adotadas são bastante variadas, e em uma mesma empresa pode haver várias escalas. Nas décadas de 60 e 70, escalas de turnos em que predominava o rodízio semanal dos horários de trabalho eram bastante freqüentes. Atualmente, em função dos estudos realizados que evidenciaram a necessidade de redução do número de jornadas noturnas, esse tipo de escala de turnos cede lugar às jornadas com rodízio mais rápido com poucos dias de trabalho noturno, jornadas extensas e irregulares, e as com trabalho em horários flexíveis. Segundo Härmä (1998), os novos padrões das escalas de trabalho em turnos seguem uma tendência mundial decorrente da introdução de novas tecnologias de produção, das flutuações das demandas, que associadas às mudanças econômicas e à globalização levariam as empresas a organizarem de forma mais eficiente suas horas de trabalho.

Knauth (1993) analisa escalas de turnos, destacando várias características que devem ser consideradas para análise. Para determinar aspectos positivos e negativos de uma escala, é necessário avaliar, pelo menos, o seguinte: o número de turnos consecutivos de trabalho, a duração de cada turno, os horários de início e final dos diversos turnos, a direção do rodízio entre os vários turnos, a regularidade dos horários de trabalho, a flexibilidade do sistema de turnos, os horários parciais ou em turno completo, a distribuição do tempo livre (pausas entre jornadas de trabalho em turnos).

Os denominados "Arranjos de Horários de Trabalho Inovadores" (Innovative Worktime Arrangements) têm sido utilizados para ajustar o tempo operacional às necessidades da força de trabalho. Também são utilizados para fazer frente a flutuações da produção, a ausências dos trabalhadores, a exigências e necessidades dos clientes. Portanto, setores tradicionais de serviços e administração cada vez mais utilizam escalas de trabalho variáveis, aumentando ou reduzindo tempos de trabalho de acordo com as necessidades. É cada vez mais freqüente, entre empresas européias, a substituição das tradicionais escalas semanais de trabalho por períodos de tempo determinados, em que os empregados são recrutados a trabalhar certos dias ou semanas de cada mês. A anualização das horas de trabalho tem sido uma dessas práticas atualmente em uso na Europa (Hornberger, 1998).

No Brasil, a Constituição Federal de 1988 determinou a redução da jornada de trabalho em turnos "ininterruptos 
de revezamento", com redução diária da jornada de trabalho a 6 horas, ou negociação coletiva (Brasil, 1988). A organização temporal do trabalho em turnos e noturno causa importantes impactos no bem-estar físico, mental e social dos trabalhadores. Usualmente, além desses há múltiplos outros fatores de risco presentes no ambiente de trabalho. São eles de variadas naturezas (física, química, biológica e organizacional) e estão relacionados a uma grande variedade de perturbações de ordem física e psicossocial. Muitas das dificuldades enfrentadas pelas organizações, como, problemas na administração de pessoal, nas comunicações, na manutenção de elevados níveis de segurança no trabalho, somam-se às dificuldades já existentes, intrínsecas ao trabalho em turnos e noturno.

Em estudo conduzido em empresa do setor gráfico em São Paulo, foi observado que a concentração de solventes no ambiente de trabalho era fator de risco adicional à saúde para os trabalhadores em turnos, aumentando os riscos de perda auditiva (Morata et al., 1997), problemas alérgicos, respiratórios e dermatológicos, bem como o risco do desenvolvimento de arritmias cardíacas.

Os aspectos associados ao trabalho em turnos e a segurança individual e pública são motivo constante de preocupação, pois existem limitações causadas pelo horário impróprio de atuação, que podem levar a graves incidentes e acidentes do trabalho (Akerstedt; Horne, 1995). Além dos numerosos acidentes causados pela sonolência excessiva de motoristas que adormecem na direção de veículos (Administração Nacional de Segurança nas Rodovias, 1999), já ocorreram grandes acidentes na indústria, como os que provocaram vazamento de material radioativo de usinas nucleares (Three Mile Island nos Estados Unidos e Chernobyl na Ucrânia) e as explosões em indústria química com vazamento de produtos tóxicos (Bophal na Índia) que ocorreram de madrugada. No entanto, além do trabalho noturno, as causas apontaram outros fatores desencadeantes, como a falta de manutenção, procedimentos inseguros, e má comunicação entre os membros das equipes que trabalhavam naqueles locais.

Uma das análises mais divulgadas na literatura sobre erros/acidentes relacionados ao trabalho em turnos foi publicada originalmente por Folkard e Monk (1979). Estes autores analisaram vários trabalhos publicados que revelavam distintas freqüências de respostas e erros ao longo do período de 24 horas; os diversos trabalhos publicados referem-se a: velocidade de responder a chamadas telefônicas, freqüência nos erros de leitura de instrumentos, freqüência de adormecimento ao volante, velocidade de tecer fios em empresa da fiação de tecidos, freqüência de ausência de resposta a sinais de alerta em maquinistas de trem e de pequenos acidentes em hospital. Em todas elas, observa-se clara tendência a piores resultados e maior número de acidentes durante a madrugada e no começo da tarde. Provavelmente, isso ocorreria em razão do pior desempenho durante o período noturno, que estaria associado à queda ou diminuição na expressão comportamental de alguns ritmos biológicos, com especial ênfase ao da temperatura corporal. Esse ritmo apresenta valores mais baixos durante a noite, concomitante ao aumento da sonolência e conseqüente queda de rendimento de algumas funções cognitivas (Monk, 1989).

Usualmente, os riscos no trabalho são analisados em função de padrões de segurança industrial estabelecidos para o trabalho diurno. Entretanto, há demonstrações dos agravos dos efeitos mais sérios de exposições ocupacionais durante os períodos não diurnos. As variações circadianas nos efeitos tóxicos levaram vários pesquisadores a questionar a segurança das exposições de acordo com os limites de tolerância aos agentes causadores de doenças ocupacionais (Lieber, 1991).

Em recente estudo apresentado no XIV International Symposium on Night and Shiftwork, em Wiesensteig, Alemanha (1999), pesquisadores canadenses e franceses observaram que os distúrbios de sono e a fadiga crônica eram os principais problemas diretamente relacionados ao trabalho em turnos de 12 horas diárias em uma refinaria canadense. A redução do número de empregados obrigava a empresa a necessitar, freqüentemente, de muitas horas extras, o que era fator de risco adicional, especialmente durante o turno diurno, resultando em excessiva fadiga, diminuição dos padrões de segurança e de confiabilidade no trabalho (Bourdouxhe et al., 1999). Os autores concluíram sua apresentação dizendo que não se deve analisar os vários aspectos das escalas de turnos fora do contexto em que se encontram, ou seja, é necessário também avaliar que tipo de tarefas são conduzidas, quais as principais cargas de trabalho, quantas pessoas realizarão o trabalho, que treinamento receberam, etc. Análises ergonômicas do trabalho auxiliam na tarefa que deve determinar o número de pessoas que comporão as equipes dos vários turnos de trabalho, nos distintos períodos do dia e da noite, em cada setor da empresa.

Em vários países europeus e nos Estados Unidos, indústrias petroquímicas trabalham 12 horas diárias. Com essa prática, pesquisadores questionam as jornadas prolongadas e seus efeitos nas manifestações de fadiga e nas 
possíveis repercussões na segurança do trabalho (Rosa, 1995).

As causas que envolvem a fadiga no trabalho são múltiplas e em geral são decorrentes das associações entre as más condições de trabalho, o desencontro entre os ritmos biológicos e os horários de trabalho. Segundo Sallinen (1997), a fadiga é mais freqüente durante à noite e em horários de trabalho que se iniciam muito cedo de manhã. Nesses últimos pode ocorrer uma privação parcial de sono por exigir que o trabalhador acorde muito cedo, dessa forma reduzindo o período de repouso. Especialmente à noite, a privação de sono causada pelas dificuldades de repouso diurno e a dessincronização dos ritmos biológicos podem reduzir significativamente os níveis de alerta dos trabalhadores e acentuar os sintomas de fadiga (Akerstedt, 1996).

\section{Fatores Individuais}

O aumento do tempo de trabalho em turnos conduz a uma cronificação de sintomas provocados pelo trabalho. De acordo com alguns autores (Koller, 1983; Haider et al., 1988), quanto maior o número de anos trabalhando em turnos, maior o número de queixas e o desenvolvimento de patologias associadas a esse tipo de esquema de trabalho. Conforme afirma Costa (1998), "a idade favorece uma intolerância progressiva, pois geralmente está associada à instabilidade de ritmos circadianos, distúrbios de sono, depressão e um declínio na capacidade física e na saúde". Assim, a idade é fator de risco adicional para o desenvolvimento de problemas de saúde e do denominado "envelhecimento funcional precoce" que pode atingir os trabalhadores em turnos ainda em idade produtiva. Monk e Folkard (1992) chamam a atenção para esse problema, pois haverá maiores contingentes de trabalhadores em turnos com idades próximas aos 50 anos ou mais, no final dessa década. Isso foi observado em estudos realizados em empresas petroquímicas privadas no Brasil, nas quais já há significativo número de pessoas com larga experiência profissional cujas idades estão ao redor, ou acima dos 50 anos. Essas pessoas, valiosas para as empresas nas quais trabalham, poderão enfrentar mais dificuldades no trabalho em turnos, especialmente se as jornadas de trabalho forem mais extensas (12 horas diárias) e tiverem menos folgas semanais.

À medida que as pessoas envelhecem modificam-se certas características dos ritmos biológicos que estão de certa forma associadas à tolerância ao trabalho em tur- nos. Alguns estudos demonstraram que as pessoas mais velhas preferem dormir mais cedo do que anteriormente o faziam. Desse modo, as pessoas tornam-se ao longo dos anos mais matutinas, o que pode dificultar a tolerância a turnos noturnos (Akerstedt; Torsvall, 1981). Segundo Wever (1981), isso poderia causar maior interferência no desempenho e mais sintomas subjetivos ao trabalhar à noite. Pessoas com hábitos e preferências vespertinos sentem-se ativas mais tarde, à noite, preferem levantar-se mais tarde de manhã, do que as pessoas matutinas. Os indivíduos com fortes tendências à vespertinidade parecem tolerar melhor o trabalho noturno do que os matutinos (Monk; Folkard, 1992).

Outra característica relacionada com a personalidade foi avaliada e associada com a tolerância ao trabalho em turnos: neuroticismo e extroversão. Observou-se que indivíduos neuróticos e introvertidos são menos capazes de suportar o trabalho em turnos e efeitos do jet-lag, do que pessoas neuróticas extrovertidas (Colquhoun; Folkard, 1978).

\section{Determinantes Sociais e Estratégias de Cunho Social e/ou Doméstico}

Os problemas sociais vividos pelos que trabalham em turnos, particularmente à noite, relacionam-se a um cotidiano essencialmente diferente do restante da comunidade como a distribuição temporal de suas atividades, como já se viu. Dependendo do esquema de turnos, podem enfrentar dificuldades de convivência com familiares e amigos, além da relativa impossibilidade de participar de cursos ou outros compromissos regulares, caminhando para o isolamento social. Diversos aspectos da vida sociofamiliar podem facilitar ou dificultar seu dia-a-dia, atuando, portanto, como fatores importantes no processo de tolerância ao regime de trabalho. Nesse contexto, cabe ressaltar os papéis sociais assumidos pelos trabalhadores, seja em casa, como cônjuge, pai/mãe, filho/a ou parente, seja fora do ambiente familiar, onde assumem papéis em relação aos amigos, clubes e atividades religiosas, entre outras. Enfim, há toda uma rede de sociabilidade cujas características tanto podem sobrecarregar o trabalhador, como, ao contrário, levá-lo a lidar melhor com o trabalho em turnos.

O gênero tem forte influência na tolerância ao trabalho em turnos, agindo mais pelas vias sociais do que por vias biológicas (Härmä, 1995; Nachreiner, 1998). Entre os que trabalham à noite, por exemplo, a reorganização da rotina 
- com intuito de acomodar no período diurno o sono e as demais atividades que compõem sua vida - é mais complexa para as mulheres, em função do papel tradicionalmente atribuído a elas quanto à casa e à família. Para elas, a realização do trabalho doméstico é prioritário em relação às demandas do sono, particularmente entre as que têm filhos, como comenta Gadbois (1981) em relação a enfermeiras que trabalham à noite. As exigências do trabalho doméstico reduzem a disponibilidade de tempo para o sono doméstico (Rotenberg et al., 2001); além disso, o tempo dedicado ao lazer também tende a ser menor entre as trabalhadoras em turnos, quando comparadas a seus colegas do sexo masculino (Knauth; Costa, 1996).

Cabe mencionar que a divisão desigual do trabalho doméstico entre homens e mulheres nem sempre se reflete em diferentes graus de tolerância ao trabalho em turnos. De fato, os estudos comparativos de homens e mulheres apresentam resultados contraditórios. Beermann e Nachreiner (1995), por exemplo, não observaram diferenças quanto aos aspectos da saúde subjetiva e a problemas psicossociais, ao passo que Oginska et al. (1993) revelaram menor duração do sono e maior freqüência de queixas de sonolência entre trabalhadores do sexo feminino. Esses dados corroboram a observação de Dirkx (1991) quanto à comparação entre amostras femininas e masculinas. Segundo o autor, quando se analisa variáveis relacionadas ao sono, os resultados são mais homogêneos do que quando se analisa outros indicadores do impacto do trabalho em turnos.

O caráter social subjacente às diferenças entre homens e mulheres fica particularmente evidente quando se analisa aspectos da vida familiar, como a presença de crianças em casa. Sabe-se, de longa data, que o ruído de crianças prejudica bastante o sono diurno em trabalhadores em turnos (Rutenfranz et al., 1989). Desse modo, ao observar menor duração do sono em trabalhadores com filhos (em relação aos demais), Anderson e Bremer (1987) atribuem o resultado ao ruído das crianças. Quanto a trabalhadores do sexo feminino, a relação presença de filhos versus sono refere-se a atividades de cuidado, como a necessidade de interromper o sono para preparar as refeições (Gadbois, 1981) ou para levar ou buscar as crianças na escola (Rotenberg, 1997), embora obviamente não se possa descartar o efeito adverso do ruído sobre o sono das trabalhadoras. Segundo Härmä (1993), a possibilidade, ou não, de contar com organizações relacionadas ao cuidado dos filhos é fator fundamental nas comparações entre trabalhadoras com e sem filhos em relação aos distúrbios do sono e queixas sobre a fadiga.
Outro aspecto das diferenças de gênero refere-se ao efeito da presença do cônjuge, como comenta Nachreiner (1998) em revisão recente sobre a tolerância ao trabalho em turnos. Segundo o autor, para as mulheres trabalhadoras, a presença do cônjuge está relacionada a maior carga de trabalho em casa, ao passo que para o trabalhador, a presença da esposa pode significar aspecto favorável à tolerância. Assim, estudos sobre a rotina doméstica entre as esposas de trabalhadores indicam um esforço delas para adaptar os horários das refeições aos turnos de trabalho do marido, adiar os serviços domésticos cujo ruído possa prejudicar seu sono diurno (Knauth; Costa, 1996) e evitar o ruído de crianças durante o dia (Bunnage, 1981). Essas precauções, que expressam o suporte doméstico por parte do cônjuge, refletem a aceitação do trabalho em turnos pelos membros da família (Monk; Folkard, 1985; Wedderburn, 1993). Como ressalta Monk (1990; 1994), quaisquer que sejam as estratégias comportamentais dos trabalhadores para lidar com o trabalho em turnos, apenas o apoio do meio social e doméstico pode garantir seu efetivo sucesso.

Entre os padrões comportamentais adotados pelos trabalhadores, as estratégias ativas no sentido de estruturar sua vida em função dos horários de trabalho contribuem fortemente para os índices de tolerância (Härmä, 1993), e são descritas como commitment to nightwork, (comprometimento com o trabalho noturno). A expressão foi usada por Folkard et al. (1978) ao compararem grupos de enfermeiras que trabalhavam quatro ou duas noites por semana, tendo constatado uma tendência, entre as profissionais do primeiro grupo, a cochilar à tarde antes da primeira noite de trabalho e a apresentar um sono mais longo entre as jornadas, o que não ocorria entre as demais enfermeiras. Essas diferenças, que os autores atribuíram a diferentes graus de comprometimento, refletiam diferentes níveis de tolerância ao trabalho, avaliados mediante ajuste dos ritmos circadianos de temperatura e da sensação de mal-estar.

O grau de organização dos horários de sono e das refeições também foi observado por Adams et al. (1986), que demonstraram a adoção desses padrões como suficientemente importante para superar as expectativas de adaptação/tolerância baseadas no tipo cronobiológico, em traços da personalidade e em algumas características do ciclo vigília-sono, com ênfase no caráter ativo das estratégias individuais. Segundo Monk e Folkard (1985), o comprometimento em relação ao horário de trabalho representa um dos mais potentes fatores 
subjacentes às diferenças entre as pessoas quanto à tolerância ao trabalho em turnos, mediando, inclusive os mais evidentes, como os relacionados ao sexo/gênero do trabalhador, provavelmente porque as possibilidades de organizar a vida tendem a diferir entre homens e mulheres, como já mencionado.

Um desdobramento dessa linha de investigação refere-se à análise da escolha do turno de trabalho como fator de tolerância ao mesmo. Em estudo com profissionais da enfermagem, Barton (1994) observou que a escolha do trabalho noturno (geralmente em função de conveniências domésticas e do adicional noturno) em oposição ao trabalho em turnos como o único disponível, tende a favorecer a tolerância ao trabalho em turnos, o que foi atribuído à maior probabilidade, entre os que trabalham à noite, de estruturar suas vidas de forma a dar conta das dificuldades associadas ao horário de trabalho.

A tolerância ao trabalho em turnos também é influenciada pela personalidade do indivíduo em termos relacionais. De acordo com Waterhouse et al. (1992), os 10\% de trabalhadores que gostam do trabalho em turnos incluem diversas pessoas com hábitos solitários nas quais as vantagens econômicas e o lazer diurno podem superar as desvantagens de um estilo de vida anormal, quando comparado às demais pessoas. Nessa mesma linha, parece ser mais fácil lidar com o trabalho em turnos quando a realização de um hobby ou a preferência por outras atividades não dependem de contatos sociais (Walker, 1985). Já entre os que se ressentem do desencontro social em relação a toda a comunidade, cabe mencionar a prática de trabalhadores em turnos alternantes de estabelecer vínculos de amizade com os próprios colegas de turma (os que estão trabalhando sempre nos mesmos horários que ele) para que possam realizar atividades sociais em seus dias de folga (Walker, 1985).

\section{O Sono em Trabalhadores em Turnos e Estratégias Relativas ao Sono para Lidar com os Horários de Trabalho}

Diante das evidências de problemas gerados pela inversão do ciclo vigília-sono e conseqüente privação do sono de trabalhadores, pode-se dizer que as estratégias individuais relacionadas aos hábitos de sono são essenciais para permitir a adaptação ao trabalho em turnos.

Tratando-se dos horários de realização dos episódios de sono em relação ao início do trabalho, é importante distinguir os diferentes horários de início do trabalho, de acordo com cada um dos turnos. Por exemplo, antes da primeira noite de trabalho, é bastante comum que as pessoas durmam à noite toda, acordando pela manhã não muito cedo, ao redor de 8 horas da manhã. Existem trabalhadores que ainda tiram um cochilo horas antes de iniciar o trabalho, que, em geral, tem a duração de 15 min a 1 hora. Já nas noites subseqüentes só resta ao trabalhador o dia para dormir. Muitos chegam em casa e dormem imediatamente, outros só vão dormir à tarde. Principalmente entre os que dormem de manhã estão os que antecipam o sono, pois tiram um cochilo poucas horas antes do início do trabalho. Dados da literatura estimam em um terço o número de trabalhadores que tiram um cochilo no final da tarde ou começo da noite (Knauth; Rutenfranz, 1981; Akerstedt, 1998). Em geral, não há ocorrência de cochilos quando os operários trabalham no turno vespertino. Já com relação ao turno matutino, verifica-se que um terço dos trabalhadores tira um cochilo à tarde, notadamente quando o início do trabalho é muito cedo, e os leva a acordar por volta das 4 horas da manhã (Akerstedt, 1998).

Menna-Barreto et al. (1993) sugeriram que a regularidade do horário de realização do episódio de sono antes do trabalho seria o fator mais importante para a adaptação ao trabalho. Entretanto, essa estratégia ainda causa polêmica, pois há pelo menos um estudo em que se verificou que a regularidade dos inícios dos episódios de sono não está relacionada à adaptação psicológica de mulheres que trabalham à noite (Rotenberg, 1997).

Alguns autores sugeriram (Minors; Waterhouse, 1981; Minors; Waterhouse, 1983) que após a divisão das 8 horas "normais" de sono em dois episódios de 4 horas cada, os ritmos biológicos estabilizariam-se, desde que um dos episódios de sono ocorresse em um horário preestabelecido do dia. Esse episódio de sono em horário fixo corresponderia ao "sono âncora" (anchor sleep) e permitiria maior tolerância dos trabalhadores ao turno noturno. Entretanto, a dificuldade de um indivíduo, que trabalhe em um esquema de turnos alternantes, em ter que realizar um episódio de sono com duração de 4 horas em horário preestabelecido pode impedir o exercício dessa estratégia.

Outros sugeriram que para compensar a privação parcial de sono, característica dos trabalhadores em turnos e noturnos, a necessidade de sono fosse suprida com a realização de episódios de sono (em especial cochilos) sempre que possível, independente do horário em que eles fossem realizados (Dinges et al., 1987; Naitoh et al., 1982). 
A irregularidade desses episódios de sono, porém, poderia provocar maior dessincronização no sistema de temporização do organismo.

A habilidade dos trabalhadores para cochilar, sejam trabalhadores submetidos a horários regulares ou irregulares de trabalho, não é a mesma para todos (Moreno, 1998; Moreno et al., 2000). Essa característica também é um dos fatores individuais que influenciam a tolerância aos horários de trabalho. Assim como as pessoas podem ser classificadas de acordo com hábitos e preferências, pode-se pensar também em "divisores do sono" (ou fragmentadores do sono) e "não-divisores do sono" (ou não-fragmentadores do sono, do inglês split sleepers e non-split sleepers).

As necessidades de sono são bastante distintas de um indivíduo para outro e, portanto, dormir de 7 a 8 horas a cada 24 horas pode ser suficiente para um trabalhador e não para outro em que a necessidade de sono é maior. Isso ocorre porque o padrão de duração do sono da espécie humana apresenta frações de sono e vigília distintas entre os indivíduos. Os que dormem mais tempo são chamados de "grandes dormidores" (costumam dormir mais de 8 horas) e os que passam pouco tempo dormindo são chamados de "pequenos dormidores" (dormem menos de 8 horas). Logo, um pequeno dormidor, poderia ser qualificado de grande vigilante, porque permanece mais tempo em vigília do que dormindo. O mesmo raciocínio pode ser utilizado para o grande dormidor, que poderia ser classificado de pequeno vigilante (Webb et al., 1970). Conclui-se, portanto, que as estratégias referentes à duração de sono dependerão das características de cada trabalhador, tanto em relação à habilidade de dividir o sono total em vários episódios, quanto em relação à duração de sono.

De acordo com Horne (1991), o sono poderia ser dividido em essencial e opcional (do inglês core and optional sleep). O sono essencial ocuparia os primeiros 3 ou 4 ciclos de sono e por ser flexível poderia ser reduzido gradativamente (20-30 min por dia durante algumas semanas). Desse modo, adultos poderiam adaptar-se a dormir diariamente cerca de 2 horas a menos sem que isso provocasse sonolência diurna. No entanto, o próprio Horne afirma que alguns indivíduos podem apresentar como estrutura de sono "natural" apenas a observada no sono essencial.

Lavie (1996) discute a proposta de Horne reforçando a idéia de que não há dúvida que pessoas que dormem muito pouco (cerca de 4 horas por dia), pois consideram o sono como grande perda de tempo, podem viver muito bem se suficientemente motivadas para isso. Todavia, os que reduzem o sono sem estímulo suficiente para permanecer em vigília, sofrem de sonolência diurna.

Stampi (1992) sugere que a espécie humana desfruta de uma habilidade endógena em adormecer várias vezes ao dia, que se expressaria em determinadas situações. Ele propôs o padrão polifásico de sono como estratégia adaptativa para situações em que os indivíduos não podem dormir o quanto gostariam, como ocorre com equipes de resgate em catástrofes como terremotos, enchentes, etc. A proposta de Stampi é que diante de uma quantidade reduzida de sono, os indivíduos adaptariamse melhor a um padrão polifásico ou semipolifásico (bifásico) de sono do que ao padrão monofásico.

Existem vários fatores que determinam se a duração total de sono ideal para um indivíduo pode ser alcançada com a realização de um único episódio de sono (padrão monofásico) ou mais de um (padrão bifásico ou polifásico). Em primeiro lugar, existe uma propensão individual que determina a habilidade de um indivíduo em dormir em diferentes horários (já citada), além disso há fatores de ordem social que determinam a necessidade de se realizar vários episódios de sono. Um estudo do sono diurno em pessoas que trabalham em turno fixo-noturno revela que o percentual de trabalhadores(as) que em geral dormem duas vezes por dia é semelhante entre homens e mulheres (44\% e 40\%, respectivamente), mas que a divisão do sono em mais de um episódio apresenta, aparentemente, um caráter diferente nas duas amostras (Rotenberg et al., 2001). Entre as mulheres, dividir o sono está associado à curta duração do sono matutino e à presença de filhos de até 10 anos, ao passo que entre os homens, o número de episódios de sono/dia não se mostra associado nem à presença de filhos, nem à duração do sono matutino. Poderse-ia especular que as diferenças entre as mulheres quanto à realização de mais de um episódio por dia são mais facilmente atribuídas a seu papel em relação ao cuidado dos filhos do que a diferenças individuais quanto aos padrões de sono. Já entre os homens, as diferenças individuais parecem mais evidentes, em função da maior possibilidade de escolher os horários de sono, já que eles não têm atribuições durante o dia. O papel social da mulher, nesse caso, parece ser um fator social de extrema importância na determinação do padrão de sono, seja polifásico ou monofásico, mas também na própria possibilidade de adotar a estratégia que possa parecer mais adequada, como sugerem os relatos dos(as) próprios(as) trabalhadores(as) (Rotenberg et al., 2001). 


\section{MEDIDAS DE INTERVENÇÃO}

\section{Nível Coletivo}

Como já se viu, o trabalho em turnos implica problemas inevitáveis, já que contraria princípios biológicos e de convivência social. Nesse sentido, as medidas para lidar com (do inglês coping) os efeitos adversos do horário de trabalho não são propriamente soluções para esses problemas, mas recomendações que visam minimizar as dificuldades dos trabalhadores quanto à saúde e ao bem-estar psicossocial. Essas medidas incluem mudanças nos esquemas temporais de trabalho e intervenções que permitem aos trabalhadores lidar com o esquema de trabalho ou tendem a reduzir suas conseqüências.

As medidas mais efetivas para contrabalançar os efeitos negativos do trabalho em turnos envolvem o desenho de esquemas de trabalho. $\mathrm{O}$ envolvimento interativo das partes envolvidas permite obter maior sucesso nas mudanças organizacionais (Gartner et al., 1998; Lillqvist et al., 1997; Smith, P.A. et al., 1998). As necessidades das empresas e os interesses de grupos de trabalhadores em turnos devem ser claramente discutidos entre as partes interessadas.

Nesse contexto, deve-se ressaltar que não há um esquema temporal "ótimo", ou seja, cada esquema apresenta vantagens e problemas do ponto de vista orgânico, psicológico ou social (Knauth, 1993). Pesquisadores da área, bem como entidades internacionais vinculadas à melhoria das condições de vida e trabalho - como a European Foundation for the Improvement of Living and Working Conditions - preconizam algumas recomendações ergonômicas que visam humanizar os esquemas de turnos (Wedderburn, 1991a).

Uma das recomendações nessa área refere-se à minimização dos turnos fixos noturnos. Caso isso não seja viável, sugere-se que a seqüência de noites trabalhadas seja a menor possível - de duas a quatro noites consecutivas (Knauth, 1993). O número de noites de trabalho está diretamente relacionado à velocidade de rotação dos turnos, ou seja, o número de dias em que o indivíduo trabalha em determinado horário. Há certa concordância entre os autores quanto à preferência por turnos de rotação rápida (Akerstedt, 1996). Levando em conta que os turnos de rotação rápida causam menos mudanças nos ritmos circadianos (Costa et al., 1994), provocam menor débito de sono (Williamson; Sanderson, 1986; Tepas; Mahan, 1989) e favorecem os contatos sociais dos trabalhadores (Knauth; Schönfelder, 1990), eles devem ser incentiva- dos em detrimento dos esquemas de rotação lenta, como os que "rodam" a cada semana, por exemplo.

Além dos problemas diretamente associados ao trabalho noturno, deve-se considerar que os turnos matutinos também tendem a provocar débitos de sono, por causa da redução do sono na(s) noite(s) que precede(m) a jornada matutina quando ela se inicia muito cedo. A afirmativa, que é tanto mais verdadeira quanto mais cedo for o início da jornada, decorre das evidências de que mesmo precisando acordar cedo no dia seguinte, as pessoas não necessariamente conseguem dormir muito mais cedo em função não apenas de pressões sociais para manter-se acordada, mas também de características dos relógios biológicos que as impedem de pegar no sono muito mais cedo que o habitual (Folkard; Barton, 1993). Outra recomendação refere-se ao sentido de rotação dos turnos. Os turnos que rodam no sentido horário, nos quais o indivíduo trabalha na seqüência manhã-tarde-noite, são mais adequados do ponto de vista dos ritmos biológicos do que os turnos que adotam sentido anti-horário, em virtude da tendência natural do sistema circadiano humano de adaptarse mais facilmente ao atraso de fase do que ao seu avanço (Monk; Folkard, 1992).

No que se refere à duração dos turnos, alguns setores adotam as "semanas curtas de trabalho" (compressed workweeks), esquemas nos quais a pessoa trabalha por um período superior a oito horas, e que resulta em uma semana com menos de cinco dias de trabalho. (Tepas, 1985). Esse tema tem merecido especial atenção nos debates sobre esquemas de trabalho, haja vista a tendência atual de adoção de turnos de 12 ou 10 horas, em substituição aos de oito horas (Axelsson et al., 1998; Lowden et al., 1998). As opiniões a respeito das vantagens e problemas decorrentes desses esquemas são controversas. No entanto, os efeitos negativos sobre a fadiga têmse revelado fatores importantes em diversos estudos, como exibe a revisão de Smith, L. et al. (1998). Seja como for, são necessárias pesquisas sistemáticas a longo prazo, de forma que se avalie os efeitos desses turnos de trabalho. De acordo com alguns autores, os turnos de 12 horas só devem ser cogitados se a natureza do trabalho e da carga de trabalho forem ajustadas para atividades de longa duração, se o esquema é planejado para minimizar o acúmulo da fadiga, se há arranjos adequados para cobrir faltas e se não houver necessidade de cumprir horas-extras (Knauth et al., 1990).

Como facilmente se pode perceber, não há como conciliar todas as recomendações, de forma que os esquemas de turnos envolvem prós e contras cuja análise é uma empreitada complexa, já que a atuação de diversas variáveis devem 
ser consideradas. Nesse contexto, cabe mencionar o desenvolvimento de programas de computador para auxiliar nas tarefas de implantação e modificação de escalas de trabalho. Com base no desenvolvimento de programas especiais, é possível planejar e construir complexas escalas de turnos que atendam aos diferentes setores da mesma empresa e que tenham incorporados critérios ergonômicos em seu desenho. Modificações nas escalas de turnos devem sempre ser acompanhadas de avaliações anteriores e posteriores às mudanças. É comum serem observadas variações na satisfação no trabalho, no sono, alerta e desempenho dos trabalhadores, após implantar novos tipos de turnos (Axelsson et al., 1998; Smith, L. et al., 1998).

De acordo com a European Foundation for the Improvement of Living and Working Conditions, um dos aspectos mais importantes no que tange às medidas de intervenção refere-se à mudança de mentalidade em relação à compensação básica pelas inconveniências do trabalho noturno (Wedderburn, 1991b). A medida tradicionalmente adotada - o pagamento extra dos trabalhadores - é considerada insuficiente para compensar o desgaste decorrente do esquema de trabalho. De fato, as medidas de compensação são objeto de profundas mudanças, havendo atualmente crescente conscientização de que pagar um extra ao indivíduo que trabalha à noite não o ajuda a dormir melhor, nem a lidar melhor com eventuais problemas familiares a que ele se expõe. Introduzida por pesquisadores holandeses nos anos 70 (Thierry et al., 1975), essa abordagem diferencia a compensação financeira das intervenções que contribuem para reduzir ou amenizar o impacto dos horários de trabalho, como, por exemplo, o atendimento médico periódico aos trabalhadores. Como comenta Wedderburn (1991b), os méritos desse modelo residem em sua flexibilidade, ou seja, a escolha das medidas depende da situação concreta e na ênfase dada à redução dos custos humanos e sociais por meio de medidas direcionadas para cada um dos custos.

Nesse contexto, a redução da "dose" de trabalho noturno é uma forma efetiva para reduzir os efeitos negativos do trabalho noturno a que o trabalhador é exposto. $\mathrm{O}$ aumento no número de folgas, a aposentadoria precoce ou transferência para turnos diurnos são algumas das formas de conseguir esse intento. Algumas dessas medidas oferecem ao trabalhador uma oportunidade para compensar problemas relacionados ao sono e à realização das tarefas domésticas, nos casos em que não haja horas-extras ou um segundo emprego (Wedderburn, 1991b).

A permissão para dormir à noite durante o turno de trabalho é uma medida que visa reduzir a fadiga e o débito de sono, que tendem a se acumular ao longo de várias noites de trabalho. A questão dos cochilos durante os turnos é controvertida, tendo sido debatida durante o XIV International Symposium on Night and Shiftwork (1999). Kazutaka Kogi, renomado pesquisador do Instituto de Ciência do Trabalho no Japão, defendeu a posição de que os cochilos durante a noite reduzem a fadiga durante e após o turno e mantêm melhores níveis de alerta ao longo da jornada, em especial a noturna. Há, entretanto, opiniões contrárias a essa prática, como a de Donald Tepas, de Connecticut, USA, pesquisador da área de trabalho em turnos por várias décadas. Segundo ele, os episódios de sono prolongado durante o trabalho poderiam atrapalhar o repouso após a jornada. No entanto, os débitos crônicos de sono acumulados ao longo das noites de trabalho podem vir a facilitar o sono "involuntário".

Algumas medidas podem ser extremamente benéficas à vida social do trabalhador, como, por exemplo, a promoção, pela empresa, de atividades de lazer e esporte durante o dia. Seminários no fim de semana podem favorecer contatos com a família. No contexto da vida social do trabalhador, cabe ressaltar que os fins de semana livres e esquemas de rotação rápida contribuem em muito para reduzir o isolamento social a que os trabalhadores em turnos são expostos.

A realização de exames médicos periódicos em trabalhadores em turnos é uma medida essencial, considerando que essa é uma população sob risco. Alguns pesquisadores enfatizam a necessidade de se ampliar a iniciativa recente de uma clínica (shiftwork clinics), planejada especificamente para atender aos trabalhadores em turnos (Monk; Folkard, 1992).

Como se viu, são várias as possibilidades de intervenção que consideram os critérios cronobiológicos. Obviamente, a adoção dessas medidas envolve negociações entre empregadores e trabalhadores nem sempre conciliáveis. Cabe lembrar o papel da legislação vigente no país quanto ao estímulo à implementação de mudanças, como a que se espera venha ocorrer no Brasil em função de nova legislação que estabelece novas regras relacionadas a fatores de risco no trabalho e suas conseqüências à saúde. De acordo com um decreto governamental recém-publicado (Brasil, 1999), grande número de agentes etiológicos de natureza ocupacional são agora reconhecidos pelo Ministério da Previdência Social, em consonância com a "Classificação Internacional de Doenças (CID-10)". Os esquemas de trabalho em turnos e noturno foram reconhecidos como agentes etiológicos de problemas do ciclo vigília-sono, o que evidencia a necessidade de ava- 
liações específicas nessas populações de trabalhadores. Essa nova legislação deve induzir as empresas a realizarem melhorias em seus esquemas de turnos a fim de prevenir afastamentos causados pelo trabalho em turnos e noturno, que resultem em disputas legais.

\section{Nível Individual}

Como já discutido, não há apenas uma única solução para o trabalho em turnos, mas uma complexa rede de fatores que podem ser modificados para facilitar a tolerância do trabalhador ao trabalho em turnos. Além das medidas coletivas citadas no item anterior, o próprio trabalhador pode adotar algumas rotinas especiais para lidar melhor com seus horários de trabalho.

De acordo com Monk e Folkard (1992), essas medidas individuais dividem-se em três áreas: sono, ritmos biológicos e situação social/doméstica do trabalhador. Em relação ao sono, os autores sugerem que hábitos regulares facilitam a tolerância ao trabalho em turnos. Rosa et al. (1990) recomendam que o trabalhador deve dormir, no mínimo, 6 horas, mas lembram que a maioria das pessoas precisa dormir mais do que isso. Dormir em quartos silenciosos, escuros e com temperatura agradável também são recomendações feitas pelos higienistas de sono e que devem ser realizadas pelo trabalhador.

Com relação aos ritmos biológicos, Monk e Folkard (1992) propõem que o cronotipo do trabalhador deve ser considerado na escolha do tipo de esquema de trabalho. Portanto, os matutinos devem optar por turnos que iniciem mais cedo, enquanto indivíduos vespertinos apresentam mais facilidade em adaptar-se ao trabalho noturno. Segundo esses mesmos autores, o peso da situação social e doméstica em promover ou dificultar a tolerância do trabalhador ao trabalho em turnos é bastante considerável. $\mathrm{O}$ apoio da família ao acompanhar o trabalhador em atividades sociais em seus horários livres, bem como manter o ambiente doméstico adequado a seu sono diurno, é fundamental para a adaptação dele ao horário de trabalho.

A prática de exercícios físicos, técnicas de relaxamento e uma dieta leve com pouca gordura e carboidratos são medidas sugeridas por Rosa et al. (1990).

A exposição à luz intensa no final da tarde também pode aumentar os níveis de alerta durante a noite dos trabalhadores. Essa medida vêm sendo muito discutida após diversas pesquisas demonstrarem que a luz intensa pode afetar a ritmicidade biológica (Rosa et al., 1990). A luz intensa altera a liberação de melatonina, hormônio pro- duzido pela glândula pineal, que, em geral, é liberado na primeira porção do sono noturno. O aumento dos níveis de melatonina induz ao sono, logo, a alteração de seus níveis também altera os níveis de sonolência de um indivíduo. A exposição à luz intensa no final da tarde reduzirá a liberação de melatonina ou atrasará sua liberação, alterando os níveis de alerta durante a noite dos indivíduos expostos (Dijk et al., 1995). Em alguns estudos de campo, com o trabalhador exposto à luz intensa combinada com o uso de óculos escuros durante o dia (sobretudo no caminho do trabalho para casa pela manhã), concluiuse que não há mais dúvidas que a exposição à luz intensa pode auxiliar trabalhadores em turnos a adaptar-se a novas rotinas de sono e vigília, porém existem ainda algumas questões que precisam de respostas, como: qual a intensidade e a duração mais adequada de exposição à luz para promover a mudança de fase nos ritmos biológicos necessária para cada tipo de turno? (Eastman et al., 1995). Por esse motivo, estudos que utilizam luz intensa tanto em laboratório, quanto em campo vêm adquirindo enorme relevância nos últimos anos.

Considerando que, nas grandes metrópoles, vive-se cada vez mais numa sociedade 24 horas, um número crescente de pessoas depara-se com as dificuldades de trabalhar em horários não diurnos e/ou fins de semana, o que torna relevante a identificação das conseqüências do trabalho em turnos e das possibilidades de amenizar os problemas por ele causados. Este artigo objetivou apresentar as múltiplas facetas dessa questão e suas possíveis soluções.

\section{NOTAS}

Este artigo corresponde à adaptação do capítulo "Tolerancia al trabajo em turnos y nocturno: uma cuestión multidimensional" (Moreno; Fischer; Rotenberg, 2002: 253-267).

1. Fonte: Censo Demográfico 2000, Fundação IBGE.

\section{REFERÊNCIAS BIBLIOGRÁFICAS}

ADAMS, J.; FOLKARD, S.; YOUNG M. Coping strategies used by nurses on nigth duty. Ergonomics. Abingdon: Taylor and Francis, v.29, p.185-196, 1986.

ADMINISTRAÇÃO NACIONAL DE SEGURANÇA NAS RODOVIAS (NHTSA)/CENTRO NACIONAL DE PESQUISAS SOBRE OS DISTÚRBIOS DO SONO DO INSTITUTO NACIONAL DO CORAÇÃO, PULMÃO E SANGUE DOS INSTITUTOS NACIONAIS DE SAÚDE (NCSDR). O ato de dirigir com sonolência e os acidentes automobilísticos. Revista da Abramet. São Paulo: Abramet, v.30, p.6-24, 1999. 
AKERSTEDT, T. Is there an optimal sleep-wake pattern in shift work? Scand J Work Environ Health. Helsinki: Scandinavian Journal Work Environment Health, v.24, s.3, p.18-27, 1998.

Wide awake at odd hours. Shiftwork, time zones and burning the midnight oil. Stockholm: Swedish Council for Work Life Research, 1996.

AKERSTEDT, T; HORNE, J. (Eds.). Work hours, sleepiness and accidents. J. Sleep Res. Oxford: Blackweel, v.4, n.2, p.1-83, 1995.

AKERSTEDT, T.; TORSVALL, L. Shiftwork: shift-dependent wellbeing and individual differences. Ergonomics. Abingdon: Taylor and Francis, v.24, p.265-273, 1981.

ANDERSON, R.M.; BREMER, D.A. Sleep duration at home and sleepiness on the job in rotating twelve-hour shift workers. Human Factors. Santa Monica: Human Factors Soc., v.29, p.477-481, 1987.

AXELSSON, J. et al. Effects of alternating 8 and 12-hour shifts on sleep, sleepiness, physical effort and performance. Scand J Work Environ Health. Helsinki: Scandinavian Journal Work Environment Health, v.24, s.3, p.62-68, 1998.

BARTON, J. Choosing to work at night: a moderating influence on individual tolerance to shift work. J. Appl. Psychology. Washington: American Psychological Assoc., v.79, p.449-454, 1994.

BEERMANN, B.; NACHREINER, F. Working shifts - different effects for women and men? Work \& Stress. Abingdon: Taylor and Francis, v.9, p.289-297, 1995.

BOURDOUXHE, M.; QUEINNEC, Y.; GUERTIN, S. The interaction between work schedule and workload: case study of 12-hour shifts in a Canadian refinery. Shiftwork International Newsletter. Karlsruhe: University Karlsruhe, v.12, n.2, p.19, 1999.

BRASIL (País). Decreto-lei no 3.048. Diário Oficial da União. Brasília: Governo Federal do Brasil. Ministério de Previdência e Seguridade Social, maio 1999.

BRASIL. Constituição Federal do Brasil. Brasília: Senado Federal, 1988.

BUNNAGE, D. Study on the consequences of shift work on social and family life. The effects of shiftwork on health, social and family life. Dublin: European Foundation for the Improvement of Living and Working Conditions, 1981.

COLQUHOUN, W.P.; FOLKARD, S. Personality differences in body temperature rhythm and their relation to its adjustment to night work. Ergonomics. Abingdon: Taylor and Francis, v.21, p.811817,1978

COSTA, G. Guidelines for the medical surveillance of shift workers. Scand J. Work Environ Health. Helsinki: Scandinavian Journal Work Environment Health, v.24, n.3, p.151-155, 1998.

The impact of shift and night work on health. Applied Ergonomics. Oxford: Elsevier Sci., v.27, n.1, p. 9-16, 1996.

COSTA, G. et al. Evaluation of a rapidly rotating shift system for tolerance of nurses to nightwork. Int. Arch. Occup. Environ. Health. New York: Springer-Verlag, v.65, p.305-311, 1994.

DIJK, D.J. et al. Light treatment for sleep disorders: consensus report. II. Basic properties of circadian physiology and sleep regulation. J. Biol. Rhythms, v.10, n.2, p.113-125, 1995.

DINGES, D.F. et al. Temporal placement of a nap for alertness: contributions of circadian phase and prior wakefulness. Sleep. Rochester: American Academy of Sleep Medicine, v.10, p.313329, 1987.

DIRKX, J. Recent research on night work for women: a review. In: SINGLETON, W.T.; DIRKX (Eds.). J. Ergonomics, health and safety: perspectives for the nineties. Leuven: Leuven University Press, p.59-75, 1991.
EASTMAN, C.I.; LIU, L.; FOGG, L.F. Circadian rhythm adaptation to simulated night shift work: effect of nocturnal bright-light duration. Sleep, v.18, n.6, p.399-407, 1995.

FISCHER, F.M et al. Shift and day workers: some features of the workers population, working conditions, accidents. A study of the metropolitan area of São Paulo, Brazil. Shiftwork International Newsletter. Pittsburg: WPIC Sleep Evaluation Center, v.12, n.1, p.115, 1995.

FOLKARD, S.; BARTON, J. Does the forbidden zone for sleep onset influence morning shift sleep duration? Ergonomics. Abingdon: Taylor and Francis, v.36, p.85-91, 1993.

FOLKARD, S.; MONK, T.H. Shiftwork and performance. Human factors. Santa Monica: Human Factors Soc., v.21, p.483-492, 1979.

FOLKARD, S.; MONK, T.H.; LOBBAN, M.C. Short and long-term adjustment of circadian rhythm in "permanent" night nurses. Ergonomics. Abingdon: Taylor and Francis, v.21, p.785-799, 1978.

GADBOIS, C. Women on night shift: interdepence of sleep and offthe-job activities. In: REINBERG, A.; VIEUX, N.; ANDLAUER, P. (Eds.). Night and shiftwork: biological and social aspects. Oxford: Pergamon Press, p.223-227, 1981.

GARTNER, J.; HÖRWEIN, K.; WAHL, S. Shiftplanassistant 3.0. A tool for innovative shift rotas. In: Proceedings of the Second European Symposium of Ergonomics. Tróia: Universidade Técnica de Lisboa, 1998.

HAIDER, M. et al. A destabilization theory of shiftwork effects. In: HEKKENS, W.TH.J.M.; KERKHOF, G.A. and RIETVELD, W.J. (Eds.). Trends in Chronobiology. Oxford: Pergamon Press, p.209217, 1988.

HÄRMÄ, M. New work times are here - are we ready? Editorial. Scand J. Work Environ Health. Helsinki: Scandinavian Journal Work Environment Health, v.24, n.3, p.3-6, 1998.

"Sleepiness and shiftwork: individual differences". $J$. Sleep Res. Oxford: Blackweel Publishing Ltd., v.4, n.2, p.57-61, 1995.

. Individual differences in tolerance to shiftwork: a review. Ergonomics. Abingdon: Taylor and Francis, v.36, p.101-109, 1993.

HORNBERGER, S. Innovative working time arrangements in production, services and administration. Proceedings of the Second European Symposium of Ergonomics. Tróia: Universidade Técnica de Lisboa, 1998.

HORNE, J.A. Dimensions to sleepiness. In: MONK, T. (Ed.). Sleep, sleepiness and performance. New York: John Wiley and Sons, 1991.

HUNTER, D. The diseases of occupations. London: Hodder and Stoughton, 1975.

KNAUTH, P. The design of shift systems. Ergonomics. Abingdon: Taylor and Francis, v.36, p.15-28, 1993.

KNAUTH, P.; COSTA, G. Psychosocial effects. In: COLQUHOUN, W.P. et al. (Eds.) Shiftwork - problems and solutions. Frankfurt: Peter Lang, p.89-112, 1996.

KNAUTH, P.; RUTENFRANZ, J. Duration of sleep related to the type of shift work. In: REINBERG, A.; VIEUX, N.; ANDLAUER P. (Eds.). Night and shiftwork: biological and social aspects. Oxford: Pergamon Press, 1981.

KNAUTH, P.; SCHÖNFELDER, E. Effects of a new shift system on the social life of shiftworkers. In: COSTA, G. et al. (Eds.). Studies in industrial organizational psychology. Shiftwork health, sleep and performance. Frankfurt: Peter Lang, v.10, p.537-545, 1990.

KNAUTH, P.; SCHÖNFELDER, E.; HORNBERGER, S. Compensation for shiftwork. Contributions to best (bulletin of 
european shiftwork topics). Dublin: European Foundation for the Improvement of Living and Working Conditions, 1990.

KOGI K. Increasing flexibility in shiftwork arrangements. Work \& Stress. Abingdon: Taylor and Francis, v.9, p.211-218, 1995.

KOLLER, M. Health risks related to shiftwork. An example of timecontingent effects of long-term stress. Int Arch Occup Environ Hlth. New York: Springer-Verlag, v.53, p.59-75, 1983.

LAVIE, P. The enchanted world of sleep. New Haven:Yale University Press, 1996. 270 p.

LENNERNÄS, M.A.C.; HAMBRAEUS, L; AKERSTEDT, T. Nutrition and shiftwork: the use of meal classification as a new tool for qualitative/quantitative evaluation of dietary intake in shiftworkers. Ergonomic. Abingdon: Taylor and Francis, v.36, n.1-3, p.247-254, 1993.

LIEBER, R.R. Trabalho em turnos e riscos químicos: o horário de trabalho como fator interveniente no efeito tóxico. 1991. Dissertação (Mestrado) - Faculdade de Saúde Pública da Universidade de São Paulo, São Paulo, 1991.

LILLQVIST, O.; HÄRMA, M.; GÄRTNER, J. Improving 5-crew shift. Työterveiset (Newsletter of the Finnish Institute of Occupational Health), Special issue - Ergonomics. Abingdon: Taylor and Francis, p.12-15, 1997.

LOWDEN, A. et al. Change from an 8-hour shift to a 12-hour shift, attitudes, sleep, sleepiness and performance. Scand J. Work Environ Health. Helsinki: Scandinavian Journal Work Environment Health, v.24, s.3, p.69-75, 1998.

MENNA-BARRETO, L. et al. Individual differences in night and continuosly-rotating shiftwork: seeking anticipatory rather than compensatory strategy. Ergonomics. Abingdon: Taylor and Francis, v.36, n.1-3, p.135-140, 1993.

MINORS D.S.; WATERHOUSE J. Does anchor sleep entrain circadian rhythms? Evidence from constant routines studies. J. Physiol. New York: Cambridge University Press, 345, p.451-467, 1983a.

Circadian rhythm amplitude - is it related to rhythm adjustment and/or workers motivation? Ergonomics. Abingdon: Taylor and Francis, v.26, p.229-241, 1983 b.

Anchor sleep as a synchronizer of rhythms on abnormal routines. Int. J. Chronobiol. Londres: John Wiley and Sons, v.7, p.165-188, 1981.

MONK, T.H. Shift work. In: KRYGER, M.H.; ROTH, T.; DEMENT, W.C. (Eds.). Principles and practice of sleep medicine. Londres: W.B.Saunders Co.,1994.

The relationship of chronobiology to sleep schedules and performance demands. Work \& Stress. Abingdon: Taylor and Francis, v.4, p.227-236, 1990

Human factors implications of shiftwork. International Rev. Ergonomics, v.2, p.111-128, 1989

MONK, T.H.; FOLKARD, S. Making shift work tolerable.Washington: Taylor and Francis, 1992. 94 p.

Individual differences in shiftwork adjustment. In: FOLKARD, S.; MONK, T.H. (Eds.). Hours of work-temporal factors in work-schedulling. Chichester: Wiley, p.227-237, 1985.

MORATA, T.M. et al. Toluene-induced hearing loss among rotogravure printing workers. Scand J. Work Environ Health. Helsinki: Scandinavian Journal Work Environment Health, v.23, p.294-303, 1997.

MORENO, C.R.C. Fragmentação do sono e adaptação ao trabalho noturno. 1998. Tese (Doutorado) - Faculdade de Saúde Pública da Universidade de São Paulo, São Paulo, 1998.

MORENO, C.R.C.; FISCHER, F.M.; ROTENBERG, L. Tolerancia al trabajo em turnos y nocturno: uma cuestión multidimensional. In:
GOLOMBEK, D. (Org.). Cronobiologia Humana. Buenos Aires: D. Universidad de Quilmes, Ediciones, 2002.

MORENO, C.R.C. et al. Two sleep patterns in night workers. In: HORNBERGER, S. et al.(Eds.). Shiftwork in the 21th Century Challenges for Research and Practice. Frankfurt: Lang, 2000.

NACHREINER, F. Individual and social determinants of shiftwork tolerance. Scand J. Work Environ Health. Helsinki: Scandinavian Journal Work Environment Health, v.24, s.3, p.35-42, 1998.

NAITOH, P.; ENGLUND, C.E.; RYMAN, DH. Restorative power of naps in designing continuous work schedules. J. Hum Ergol. Tokyo: University of Tokyo Press, v.11, p.259-278, 1982.

OGINSKA, H.; POKORSKI, J.; OGINSKI, A. Gender, ageing, and shiftwork intolerance. Ergonomics. Abingdon: Taylor and Francis, v.36, p.161-168, 1993.

PRESSER, H.B. Toward a 24-hour economy. Science, n.284, p.1778$1779,1999$.

ROSA, R.R. Extended workshifts and excessive fatigue. J. Sleep Res. Oxford: Blackwell Publishing, v.4, p. 51-56, 1995.

ROSA, R.R. et al. Intervention factors for promoting adjustment to nightwork and shiftwork. Occup. Med., n.5, p.391-415, 1990.

ROTENBERG, L. Trabalhando de noite e dormindo de dia - regularidade do sono e adaptação psicológica em operárias do turno noturno. 1997. Tese (Doutorado) - Instituto de Psicologia da USP, São Paulo, 1997.

ROTENBERG, L. et al. Gênero e trabalho noturno: sono, cotidiano e vivências de quem troca a noite pelo dia. Cadernos de Saúde Pública, v.17, n.3, p.639-649, 2001.

RUTENFRANZ, J.; KNAUTH, P.; FISCHER, F.M. Trabalho em turnos e noturno. São Paulo: Hucitec, 1989. 135 p.

SALLINEN, M. Fatigue in shift work. Työterveiset, Special Issue. Ergonomics. Abingdon: Taylor and Francis, p. 9-11, 1997.

SMITH, L. et al. Work shift duration: a review comparing eight hour and 12 hour shift systems. Occup Environ Med. Londres: British Med Journal Publ Group, v.55, p. 217-229, 1998.

SMITH, P.A. et al. Change from slowly rotating 8 hour shifts to rapidly rotating 8 hour and 12 -hour shifts using participative shift roster design. Scand J. Work Environ Health. Helsinki: Scandinavian Journal Work Environment Health, v.24, s.3, p.55-61, 1998.

STAMPI, C. Why we nap: evolution, chronobiology and functions of polyphasic and ultrashort sleep. Boston: Birkhauser, 1992.

TEPAS, D.I. Flextime, compressed workweeks and other alternative work schedules. In: FOLKARD, S.; MONK, T.H. (Eds.). Hours of work-temporal factors in work-schedulling. Chichester: Wiley, p.147-164, 1985.

TEPAS, D.I.; MAHAN, R.P. The many meanings of sleep. Work \& Stress. Abingdon: Taylor and Francis, v.3, p.93-102, 1989.

THIERRY, H. K; HOOLWERF, G.; DRENTH, P.J.D. Attitudes of permanent day and shift workers towards shiftwork - a field study. In: COULQUHOUN, W.P. et al. (Eds.). Experimental studies of shiftwork. Opladen: Westdeutscher Verlag, p.213-231, 1975.

WALKER, J. Social problems of shiftwork. In: FOLKARD, S.; MONK, T.H. (Eds.). Hours of work-temporal factors in work-scheduling. Chichester: Wiley, p.211-225, 1985.

WATERHOUSE, J.M.; FOLKARD, S.; MINORS, D.S. Shiftwork, health and safety - an overview of the scientific literature 19781990. HSE Contract Research Report n.31, 1992.

WEBB, W.B.; FRIEL, J. Characteristics of natural long and short sleepers: a preliminary report. Psychol. Res. Berlim: SpringerVerlag, v.27, n.1, p.63-66, 1970. 
WEDDERBURN, A. La semaine de travail comprimee. Dublin: Foundation Europenne pour l'Amelioration des Conditions de Vie et de Travail, 1997.

Social and family factos in shift design. Dublin: European Foundation for the Improvement of Living and Working Conditions, 1993.

Guidelines for shiftworkers. European Foundation for the Improvement of Living and Working Conditions, Dublin, 1991a. $56 \mathrm{p}$.

. Compensation for shiftwork. Dublin: European Foundation for the Improvement of Living and Working Conditions, 1991b.

WEVER R. On varying work-sleep schedules: the biological rhythm perspective. In: JOHNSON, L.C. et al. (Eds.). Biological rhythms, sleep and shiftwork. Advances in Sleep Research. New York: Spectrum, v.7, p.36-60, 1981.
WILLIAMSON, A.M.; SANDERSON, J.W. Changing the speed of shift rotation: a field study. Ergonomics. Abingdon: Taylor and Francis, v.29, p.1.085-1.096, 1986.

Claudia Roberta de Castro Moreno: Bióloga, Pesquisadora do Departamento de Psicobiologia da Escola Paulista de Medicina, UnifespSP (crmoreno@psicobio.epm.br).

Frida Marina Fischer: Bióloga, Professora do Departamento de Saúde Ambiental, Faculdade de Saúde Pública da USP (fmfische@usp.br)

Lúcia Rotenberg: Bióloga, Pesquisadora do Departamento de Biologia do Instituto Oswaldo Cruz, Fiocruz-RJ (rotenber@ioc.fiocruz.br). 